\title{
An Experience-Sampling Study of Depressive Symptoms and Their Social Context
}

\author{
Leslie H. Brown, PhD, * Timothy Strauman, PhD, $\dagger$ Neus Barrantes-Vidal, PhD, $\ddagger \$ / / \mathbb{I}$ \\ Paul J. Silvia, PhD,// and Thomas R. Kwapil, PhD//
}

\begin{abstract}
Both clinical and subclinical depression are associated with social impairment; however, few studies have examined the impact of social contact in the daily lives of people with depressive symptoms. The current study used the experience-sampling methodology to examine associations between depressive symptoms, social contact, and daily life impairment in 197 young adults. Depressive symptoms were associated with increased isolation, negative affect, anhedonia, and physical symptoms, decreased positive affect, and social and cognitive impairment in daily life. For people with more depressive symptoms, being with social partners who were perceived as close was associated with greater decreases in negative affect, as well as increases in positive affect. Ironically, participants with depressive symptoms reported spending less time with people whom they perceived as close, minimizing the protective effects of socializing. These results suggest that people experiencing depressive symptoms may be especially sensitive to the nature of social interactions.
\end{abstract}

Key Words: Depression, experience-sampling method, ecological momentary assessment.

(J Nerv Ment Dis 2011;199: 403-409)

$\mathrm{D}$ epressive disorders are among the most common psychological diagnoses, with lifetime prevalence estimates ranging from $16 \%$ to $31 \%$ for women and $11 \%$ to $18 \%$ for men (American Psychological Association, 2004). A substantial body of research indicates that clinical and subclinical levels of depression are associated with impaired social functioning and decrements in perceived social support. For example, low self-reported social support is highly correlated with the onset and outcome of depressive episodes (Billings and Moos, 1984). This is especially problematic because perceived social support appears to be protective against stressful life events (Cohen and Wills, 1985 ) and, thus, those with depressive symptoms may be deprived of a powerful buffer against life stress. Deficits in social functioning correspond to the severity of depressive symptoms and may improve when depression remits (e.g., Airaksinen et al., 2006). Furthermore, cognitive-behavioral therapy is associated with improvements in depression that co-occur with improvements in social functioning (Scott et al., 2000). Not surprisingly, treatments for depression, such as Interpersonal Psychotherapy (e.g., Klerman et al., 1984), often focus on the expression and treatment of depression within the social environment.

A growing body of literature suggests that people with clinical and subclinical depression may be sensitive to the quality and closeness of social interactions. A recent study of nondepressed under-

*University of Pittsburgh School of Medicine, Pittsburgh, PA; †Duke University, Durham, NC; †Universitat Autònoma de Barcelona, Barcelona, Spain; §Sant Pere Claver-Fundació Sanitària, Barcelona, Spain; |University of North Carolina at Greensboro, Greensboro, NC; IInstituto de Salud Carlos III, Centro de Investigación Biomédica en Red de Salud Mental, CIBERSAM, Barcelona, Spain.

Leslie Brown was supported by a National Science Foundation Graduate Research.

Send reprint requests to Leslie H. Brown, University of Pittsburgh School of

Medicine, Sterling Plaza Suite 200, 201 N Craig St, Pittsburgh, PA 15213.

E-mail: brownlh@upmc.edu.

Copyright (C) 2011 by Lippincott Williams \& Wilkins

ISSN: 0022-3018/11/19906-0403

DOI: $10.1097 / \mathrm{NMD} .0 \mathrm{~b} 013 \mathrm{e} 31821 \mathrm{~cd} 24 \mathrm{~b}$ graduates (Panzarella et al., 2006) found that people who received more adaptive inferential feedback (i.e., feedback in which friends and family reframe life events in a more adaptive or positive light) experienced improvements in depressive symptoms, even after controlling for the effects of general social support and stressful life events. For example, a person with depression might benefit from having a friend describe his or her recent job loss as an opportunity for a "fresh start," rather than as a tragedy. Therefore, it is possible that specific types of social interactions common among close family and friends may be more beneficial to mood than to social contact per se. In other words, not all types of social interactions are created equal, and the type of support and feedback provided during a close social interaction could influence mood differently. Examining differences in affect during close versus nonclose social encounters may provide a point of entry to better understand how social context impacts the lives of people with depressive symptoms.

\section{Depression as a Dimensional Construct}

A growing body of research supports depression as a dimensional construct, with diagnostic categories representing the most severe manifestations on this continuum (Krueger and Markon, 2006). For example, Hankin et al. (2005) examined self-reported depressive symptoms using taxometric procedures and found that depression was distributed continuously in community samples. Furthermore, subclinical manifestations of mood disorders are associated with impairment in functioning and reduced quality of life (Judd et al., 1996; Kessler et al., 1997), in addition to being precursors to the development of clinical depression (Brown et al., 1986; Lewinsohn et al., 1988). In the present study, we used the term depressive symptoms to refer to this broader continuum of clinical and subclinical depression and to avoid confusion with categorical diagnoses.

\section{The Experience-Sampling Methodology in the Study of Depressive Symptoms}

Researchers have recently begun using experience-sampling methodology (ESM) to explore the daily life experiences of individuals with psychopathology and the context in which their experiences occur. ESM is a within-day self-assessment technique in which participants are prompted at random intervals to report on their current experiences. ESM offers several advantages over traditional data collection procedures (e.g., Csikszentmihalyi and Larson, 1987; Reis and Gable, 2000). Specifically, ESM a) repeatedly assesses participants in their normal daily environment, thereby enhancing ecological validity, b) assesses the participants' experiences in the moment, thereby minimizing retrospective bias, and c) allows for an examination of the context of experiences.

Several studies have used ESM to examine depression in daily life. Wang et al. (2004) found increased levels of somatic symptoms in the daily lives of depressed compared with those of nondepressed office workers. Peeters et al. (2006) reported that relative to nondepressed individuals, depressed individuals exhibited overall less positive affect (PA) and more negative affect (NA), with increasing PA levels during the daytime and a more pronounced diurnal NA rhythm. Rusting and Larsen (1998) reported significant associations between 
the evening-worse depressive pattern and neurotic features, scores on depression and anxiety measures, and hopelessness. In a study of the daily lives of older adults, major depression was associated with decreased PA, whereas dysthymia was associated with increased NA (Chepenik et al., 2006). A study conducted by Barge-Schaapveld et al. (1999) found that depressed people reported higher NA, lower PA, more physical complaints, and less enjoyment of the current activity than did nondepressed individuals. However, none of these studies examined the expression of depression across a broad range of clinical and subclinical severity, nor did they specifically examine the effects of social contact on the expression of depressive symptoms.

\section{Goals and Hypotheses}

The goals of the current study were to examine the associations between self-reported depressive symptoms and reports of affect, cognition, activities, and social functioning in daily life. Furthermore, we sought to examine the extent to which social contact and closeness of social contact impacted the expression of depressive symptoms in daily life. Specifically, we hypothesized that depressive symptoms would be associated with increased NA and diminished PA, as well as reports of physical symptoms and cognitive impairment in daily life. Within the social domain, we predicted that depressive symptoms would be associated with increased social isolation, more social distance and disengagement from others, greater preference to be alone when with others, and reports that they were alone because others do not want to be with them. In addition, we predicted that depressive symptoms would moderate the relations of social contact and affect in daily life. In general, we expected that the participants would experience more PA and less NA when with others. However, we predicted that these relations would be stronger in participants with higher selfrated depressive symptoms, consistent with reactivity to social cues associated with sociotropy (Beck, 1983; Coyne and Whiffen, 1995); although, as noted, we expected the main effect that participants with higher depressive symptoms would report more NA and less PA in daily life than would their peers with lower depressive symptoms. In other words, we expected that the improvement in affect when with others would be greater in participants with higher depressive ratings. Likewise, we hypothesized that closer social contacts would be associated with greater PA and lower NA and that these relations would be stronger for participants with depressive symptoms (i.e., depressive symptoms would moderate the association between self-reported closeness and affect).

Consistent with our interpretation that social closeness has an especially beneficial effect for people who report depressive symptoms, we predicted that PA measured at the previous time point would predict PA at the current time point and that, over and above the variance accounted for by previous PA, social closeness measured at the previous time point would predict PA at the current time point. However, we also expected that social closeness at the previous time point would predict closeness at the current time point but that PA measured at the previous time point would not predict closeness over and above the closeness at the previous time point. Therefore, we predicted that closeness would predict subsequent affect but that affect would not predict subsequent closeness.

Given that depression is more commonly reported in women, we also examined the effect of sex on daily life experiences and the extent to which the association between depressive symptoms and daily life experiences was moderated by sex (i.e., a sex $\times$ depressive symptoms interaction).

\section{METHODS}

\section{Participants}

The sample consisted of 150 women and 47 men enrolled at University of North Carolina at Greensboro. The sample was $72 \%$
Caucasian, 25\% African-American, 1\% Hispanic, 1\% Asian-Pacific islander, and $1 \%$ unidentified. The mean (SD) age was 19.4 (1.9) years. The distributions of sex, ethnicity, and age were consistent with university demographics.

\section{Materials and Procedures}

The participants completed a brief demographic questionnaire and the Beck Depression Inventory (BDI; Beck et al., 1961) as part of mass screening sessions. The 21 -item BDI assesses a variety of depressive symptoms across a range of severity. The coefficient $\alpha$ was 0.90 for the BDI in the present sample. BDI scores ranged from 0 to 36 with a mean (SD) of 7.9 (7.7). Although we did not specifically recruit individuals with clinically significant levels of depressive symptoms, $32 \%$ of the participants scored in the mildly to severely depressed range, including three participants in the severe range $(>30)$. Although estimated rates of subclinical and clinical depression in late adolescence vary, these rates are consistent with those of larger, nonclinical samples of adolescents (e.g., Fergusson et al., 2007).

ESM data were collected on Personal Digital Assistants (PDAs) using iESP software (Intel, 2004). The 36-item ESM questionnaire inquired about affect, social contact, cognitions, and activities at the time of the signal (Table 1). Following Myin-Germeys et al. (2001), summary indices were computed for PA (coefficient $\alpha=0.90$ ), NA (0.90), social distance (0.90), and thought impairment $(0.83)$. It is notable that, unlike the BDI, the ESM questionnaire was not a measure of depression but rather was a measure of the participants' social, emotional, cognitive, and behavioral functioning in daily life.

The participants attended a group information session in which experimenters provided PDAs and described the procedures. After being assigned a PDA and being provided verbal instructions on its use during the initial session, the participants were asked to complete a practice questionnaire to ensure familiarity with study procedures. Before the participants left the session, they were provided with a written summary of the study instructions and contact information in the event that they experienced problems with the procedures.

The PDAs signaled the participants, administered the questionnaires, and time-stamped and recorded the responses. The participants were signaled randomly to complete the questionnaire eight times between noon and midnight for 7 days. The participants were also asked to return to the laboratory on days 2 and 4 of the study to allow the investigators to download their current data. These visits decreased the likelihood of data loss resulting from lost or defective PDAs and increased the likelihood of participants regularly completing the protocols. The participants had up to 5 minutes to initiate their responses following the signal and up to 3 minutes to complete each subsequent question. After these time intervals (or the completion of a questionnaire), the PDA would turn off and would not reactivate until the next signal. The questionnaires required about 2 minutes to complete.

\section{Statistical Analyses}

ESM data have a hierarchical structure in which ESM ratings (level 1 data) are nested within participants (level 2 data). Hierarchical linear modeling provides a more appropriate method than unilevel regression for analyzing nested data, in part because a participant's scores are not aggregated (Schwartz and Stone, 1998). The initial analyses assessed the independent effects of the level 2 predictors (BDI score, sex, BDI $\times$ sex interaction) on level 1 dependent measures (ESM ratings in daily life). Additional analyses examined the cross-level interactions of the level 1 ESM variables (e.g., PA and social contact) with the level 2 ratings. Cross-level interactions (or slopes-as-outcomes effects, as they are sometimes called; Kreft and de Leeuw, 1998) test whether level 1 associations (e.g., the association between social contact and PA in daily life) vary as a function of level 2 variables. In other words, cross-level interactions test, for example, 


\section{TABLE 1. ESM Questionnaire and Summary Indices}

\section{Questionnaire}

1. My thoughts are clear right now.

2. I have trouble concentrating right now.

3. My thoughts are suspicious right now.

4. I feel happy right now

5. I feel uncertain right now.

6. I feel lonely right now.

7. I feel relaxed right now.

8. I feel guilty right now.

9. I feel satisfied right now.

10. I feel anxious right now.

11. I feel sad right now.

12. I feel enthusiastic right now.

13. I feel irritable right now.

14. I like what I am doing right now.

15. It takes a lot of effort to do this activity.

16. I have the ability to do this activity.

17. I would prefer to do something else right now.

18. I feel tired right now.

19. I feel hungry right now.

20. I don't feel well right now.

21. Are you alone at this time?

Yes

No

If not alone (answered "No" to Question 21):

22 . How many people with you are male?

$\begin{array}{ccccc}0 & 1 & 2 & 3 & 4+ \\ 23 . \text { How many people with you are female? } & & \\ 0 & 1 & 2 & 3 & 4+\end{array}$

24. I am with (check all that apply):

1. Significant other

2. Family

3. Friend

4. Classmate

5. Co-worker

6. Stranger

7. Other

25. I like this person (these people).

26. My time with this person (these people) is important to me.

27. We are interacting together.

28. I feel close to this person (these people).

29. Right now I would prefer to be alone.

If alone (answered "Yes" to Question 21):

30. Right now, I enjoy being alone.

31. Being alone right now is my choice.

32. I am alone right now because people do not want to be with me.

33. Right now, I would prefer to be with other people.

To be answered by all participants:

34. Since the last beep, the most important thing that happened to me was pleasant.

35. The most important thing that happened to me involved being with other people.

Yes

36. This beep disturbed me.

Summary Indices ${ }^{\mathrm{a}}$

Positive affect: $4,7,9$, and 12

Negative affect: $5,6,8,10$, and 11

Social distance: 25r, 26r, 27r, 28r, and 29

Thought impairment: $1 \mathrm{r}$ and 2

ESM indicates experience-sampling methodology.

${ }^{a}$ Indices are computed as the mean of the items indicated. The letter $r$ indicates that an item is reverse-scored. whether the relation of PA and reported closeness differs for people higher in versus those lower in depression. If a level 2 predictor is significant, then it explains the variability in the within-person slopes. These analyses also evaluated the strength of the relation of the level 1 predictor and criterion independent of the level 2 variables. These values provided an effective test of the validity of the assessment of daily experiences, although they were not necessarily directly related to hypotheses regarding depression. For all analyses, the level 2 predictors, BDI score and sex, were entered simultaneously at the first step, and the interaction term was entered at the second step. The multilevel analyses were conducted Hierarchical Linear and Nonlinear Modeling 6 (HLM 6, Lincoln, IL; Raudenbush et al., 2004). Consistent with the recommendations of Hox (2002), the level 1 predictors were group mean-centered (i.e., centered within individuals), and the level 2 predictors were grand mean-centered.

\section{RESULTS}

Participants completed an average (SD) of 41 (11) ESM questionnaires during the week. Sex was not associated with the number of completed ESM questionnaires $(r=0.07)$, although BDI scores had a modest inverse correlation with the number of completed questionnaires $(r=-0.19, p<0.01)$. There was a modest correlation between BDI score and sex $(r=0.21, p<0.01)$ indicating that BDI scores were higher on average in women than in men.

Table 2 presents the associations of depressive symptoms (BDI score) with reports of affect and physical functioning in daily life. Depressive symptoms were associated with diminished PA, increased NA, and reports that important events were perceived as less pleasant. Depressive symptoms were also associated with reports of impaired physical functioning.

Table 3 presents the associations of depressive symptoms with the reports of social contact and functioning in daily life. Higher rates of depressive symptoms were associated with a greater likelihood of being alone at the time of the signal. When participants reported being with others, depressive symptoms were associated with increased social distance, including lower ratings of liking and feeling close to others. Depressive symptoms were associated with a stronger preference to be alone when with other people at the time of the signal. When the participants were alone, depressive symptoms were associated with an increased desire to be with others and with the belief that they were alone because others do not want to be with them.

Depressive symptoms were associated with increased impairment in cognitions and daily activities (Table 4). Specifically, depressive symptoms were associated with impaired concentration and clarity of thought and increased suspiciousness. In terms of daily activities, depressive symptoms were associated with less enthusiasm and enjoyment of current activity and a greater preference to do something else other than the current activity.

There were significant sex differences independent of the effects of depression in a number of analyses. Women reported greater NA, less PA, and more thought and physical complaints, over and above the effects of depressive symptoms. However, only one of the sex $\times$ depressive symptoms interaction coefficients was statistically significant, indicating that, in general, the relation of depressive symptoms with experiences in daily life did not differ for men and women.

To examine the effect of social contact on the expression of depressive symptoms in daily life, we conducted two sets of crosslevel interaction analyses. These analyses looked at the impact of social contact at the time of the signal and the report of feeling close to people when one was with others on reports of PA, NA, not feeling well, and having the ability to do the current activity. Table 5 presents the cross-level interaction analyses for social contact and closeness. It is notable that none of the sex $\times$ depressive symptoms interactions 
TABLE 2. Relation of Depressive Symptoms and Sex With Experiences in Daily Life: Affect and Physical Functioning

\section{Level 2 Predictors}

\begin{tabular}{lccr}
\cline { 2 - 4 } Level 1 Criterion & Step 1: Sex, $\boldsymbol{\gamma}_{\mathbf{0 1}}(\boldsymbol{d} \boldsymbol{f}=\mathbf{1 9 4})$ & Step 1: Depression, $\boldsymbol{\gamma}_{\mathbf{0 2}}(\boldsymbol{d} \boldsymbol{f}=\mathbf{1 9 4})$ & Step 2: Sex $\times$ Depression, $\boldsymbol{\gamma}_{\mathbf{0 3}}(\boldsymbol{d} \boldsymbol{f}=\mathbf{1 9 3})$ \\
\hline Positive affect index & $-0.098(\mathrm{SE}, 0.049)^{*}$ & $-0.308(\mathrm{SE}, 0.047)^{* * *}$ & $-0.042(\mathrm{SE}, 0.081)$ \\
Negative affect index & $0.109(\mathrm{SE}, 0.049)^{*}$ & $0.378(\mathrm{SE}, 0.051)^{* * *}$ & $-0.083(\mathrm{SE}, 0.062)$ \\
Event pleasantness & $-0.156(\mathrm{SE}, 0.059)^{* *}$ & $-0.162(\mathrm{SE}, 0.068)^{*}$ & $0.012(\mathrm{SE}, 0.077)$ \\
Don't feel well & $0.217(\mathrm{SE}, 0.079)^{* *}$ & $0.425(\mathrm{SE}, 0.085)^{* * *}$ & $-0.266(\mathrm{SE}, 0.126)^{*}$ \\
Tired & $0.242(\mathrm{SE}, 0.071)^{* *}$ & $0.291(\mathrm{SE}, 0.075)^{* * *}$ & $-0.056(\mathrm{SE}, 0.117)$ \\
\hline
\end{tabular}

Raw multilevel regression coefficients indicating the relation of the level 2 predictors with the level 1 (daily life experience) criteria. For effects of sex on daily life experiences, positive coefficients indicate that women exceeded men, and negative coefficients indicate that men exceeded women.

$* p<0.05$.

$* * p<0.01$.

$* * * p<0.001$.

was significant, so they were omitted from the table for the sake of parsimony. The report of being with other people in daily life was significantly associated with increased PA, decreased NA, and decreased reports of not feeling well. However, these associations were not moderated by sex or depressive symptoms. Therefore, people higher in depressive symptoms, like other participants, experienced a boost in mood when with others compared with when alone; nevertheless, those with depressive symptoms had an overall lower mood across situations.

The reports of greater social closeness in the moment had the beneficial effects of increased PA and the belief that one has the ability to complete the current activity, as well as decreased reports of NA and not feeling well. There were also significant cross-level interactions of depressive symptoms with the association of closeness with PA, NA, and not feeling well, suggesting that the closeness of current social interactions impacted the expression of depression in daily life. As seen in Figure 1, people reporting higher levels of depressive symptoms received more benefit from social closeness in daily life than did the participants lower in depression. Nevertheless, social closeness did not completely ameliorate the impairment experienced by people reporting higher depressive symptoms.

To examine the temporal relations of social closeness and affect (i.e., to what extent did closeness at one signal predict affect at a subsequent signal, and vice versa), we conducted a series of post hoc timelagged analyses. Consistent with our initial interpretation that social closeness has an especially beneficial effect for people who report depressive symptoms, we expected that PA measured at the previous time point would predict PA at the current time point and that, over and above the variance accounted for by previous PA, social closeness measured at the previous time point would predict PA at the current time point. However, we also predicted that social closeness at the previous time point would predict closeness at the current time point but that PA measured at the previous time point would not predict closeness over and above closeness at the previous time point. In other words, we predicted that closeness would predict subsequent PA but that PA would not predict subsequent closeness. We predicted a similar pattern with closeness and NA.

As seen in Figure 2, these hypotheses were supported. Specifically, PA measured at the previous time point predicted PA at the current time point (as seen in path [a]) and, over and above the variance accounted for by previous PA, social closeness measured at the previous time point predicted PA at the current time point (path [b]). As seen in path (c), previous closeness predicted current closeness, but previous PA did not account for additional variance in current closeness (path [d]). The same pattern emerged in the predictions involving NA (see paths $[\mathrm{e}]$ to $[\mathrm{h}]$ ). Note that the valence of the relation in path (f) is reversed from path (b) because previous closeness predicted increased PA but decreased NA. Therefore, social closeness at the previous time point significantly predicted affect at the current assessment (over and above the influence of affect at the previous time point). However, affect

TABLE 3. Relation of Depressive Symptoms With Experiences in Daily Life: Social Contact and Functioning

\section{Level 2 Predictors}

Level 1 Criterion

Step 1: Sex, $\gamma_{01}(d f=194) \quad$ Step 1: Depression, $\gamma_{02}(d f=194) \quad$ Step 2: Sex $\times$ Depression, $\gamma_{03}(d f=193)$

\begin{tabular}{|c|c|c|c|}
\hline Alone at signal $(1=$ alone; $2=$ with others $)$ & $-0.001(\mathrm{SE}, 0.011)$ & $-0.032(\mathrm{SE}, 0.012)^{* *}$ & 0.019 (SE, 0.015) \\
\hline \multicolumn{4}{|l|}{ When with others: } \\
\hline Social Distance Index & $-0.059(\mathrm{SE}, 0.048)$ & $0.171(\mathrm{SE}, 0.059)^{* *}$ & 0.005 (SE, 0.062) \\
\hline Like person & 0.074 (SE, 0.047) & $-0.142(\mathrm{SE}, 0.060)^{*}$ & $-0.042(\mathrm{SE}, 0.065)$ \\
\hline Time with person is important to me & $0.105(\mathrm{SE}, 0.065)$ & $-0.121(\mathrm{SE}, 0.071)$ & $0.026(\mathrm{SE}, 0.088)$ \\
\hline Close to other & $0.137(\mathrm{SE}, 0.069)^{*}$ & $-0.171(\mathrm{SE}, 0.071)^{*}$ & $-0.012(\mathrm{SE}, 0.081)$ \\
\hline Prefer to be alone & $0.052(\mathrm{SE}, 0.066)$ & $0.260(\mathrm{SE}, 0.067)^{* * *}$ & $-0.003(\mathrm{SE}, 0.093)$ \\
\hline \multicolumn{4}{|l|}{ When alone: } \\
\hline Alone because not wanted & $0.024(\mathrm{SE}, 0.048)$ & $0.200(\mathrm{SE}, 0.066)^{* *}$ & $-0.080(\mathrm{SE}, 0.071)$ \\
\hline Prefer to be with others & $0.012(\mathrm{SE}, 0.088)$ & $0.236(\mathrm{SE}, 0.077)^{* *}$ & $-0.212(\mathrm{SE}, 0.119)$ \\
\hline
\end{tabular}

Raw multilevel regression coefficients indicating the relation of the level 2 predictors with level 1 (daily life experience) criteria. For effects of sex on daily life experiences, positive coefficients indicate that women exceeded men, and negative coefficients indicate that men exceeded women.

$* p<0.05$.

$* * p<0.01$.

$* * * p<0.001$. 
TABLE 4. Relation of Depressive Symptoms With Experiences in Daily Life: Cognition and Daily Activities

\section{Level 2 Predictors}

\begin{tabular}{|c|c|c|c|}
\hline Level 1 Criterion & Step 1: Sex, $\gamma_{01}(d f=194)$ & Step 1: Depression, $\gamma_{02}(d f=194)$ & Step 2: Sex $\times$ Depression, $\gamma_{03}(d f=193)$ \\
\hline \multicolumn{4}{|l|}{ Cognition } \\
\hline Thought Impairment Index & $0.140(\mathrm{SE}, 0.063)^{*}$ & $0.387(\mathrm{SE}, 0.055)^{* * *}$ & $-0.040(\mathrm{SE}, 0.091)$ \\
\hline Clear thoughts & $-0.134(\mathrm{SE}, 0.071)$ & $-0.397(\mathrm{SE}, 0.066)^{* * *}$ & $-0.031(\mathrm{SE}, 0.085)$ \\
\hline Suspicious thoughts & 0.007 (SE, 0.062) & $0.202(\mathrm{SE}, 0.067)^{* *}$ & $-0.155(\mathrm{SE}, 0.078)$ \\
\hline \multicolumn{4}{|l|}{ Current activity } \\
\hline Like activity & $-0.088(\mathrm{SE}, 0.049)$ & $-0.215(\mathrm{SE}, 0.052)^{* * *}$ & $0.061(\mathrm{SE}, 0.063)$ \\
\hline Have ability & $-0.060(\mathrm{SE}, 0.045)$ & $-0.128(\mathrm{SE}, 0.051)^{*}$ & $0.038(\mathrm{SE}, 0.056)$ \\
\hline Prefer another activity & $0.172(\mathrm{SE}, 0.051)^{* *}$ & $0.288(\mathrm{SE}, 0.062)^{* * *}$ & $-0.065(\mathrm{SE}, 0.061)$ \\
\hline
\end{tabular}

at the previous time point does not predict closeness at the current assessment, over and above the effect of closeness at the previous time point).

\section{DISCUSSION}

There is an extensive literature reporting on the reciprocal effects of depression and social functioning (Barnett and Gotlib, 1988; Coyne and Whiffen, 1995). That literature documents a tendency for individuals with subclinical and clinical levels of depressive symptoms to report behavioral difficulties and increased distress associated with interpersonal interactions. However, to our knowledge, the present study is the first to examine the impact of social contact and closeness on the expression of depressive symptoms in the moment.

As hypothesized, depressive symptoms were associated with social isolation and decreased quality of relationships. Furthermore, the time-lagged analyses indicated that social closeness at the previous assessment predicted improved affect at the current assessment over and above the affect ratings at the previous assessment. When the participants were with others, the depressive symptoms were associated with increased social distance and a stronger preference to be alone. When the participants were alone, on the other hand, depressive symptoms were associated with an increased desire to be with others and attributions of not being wanted. In other words, depressive symptoms were generally associated with discomfort when with others and with feeling rejected when alone. However, the impact of depression in the moment appeared to be reduced by being with others described as close. Ironically, however, the participants with depressive symptoms tended to spend less time with others and report less closeness, thus depriving them of this uplift in mood.

This study had limitations that should be addressed in future studies of depression and social contact. First of all, we included only the BDI to measure depressive symptoms; therefore, conclusions on how these findings can be applied to those with clinical disorders should be viewed as preliminary. Future studies should include multiple measures and methods for assessing depression across a continuum of severity. Another limitation of the present study is that a floor effect (for example, low negative affect in nondepressed participants) could account for the reported interactions between level 2 variables and level 1 predictors and outcomes.

These findings suggest that the people's report of the quality and nature of social relationships, rather than of the mere presence

TABLE 5. Cross-Level Interactions of Social Contact and Closeness With Affect and Functioning

\begin{tabular}{|c|c|c|c|}
\hline \multirow[b]{2}{*}{ Level 1 Criterion } & Level 1 Predictor & \multicolumn{2}{|c|}{ Level 2 Predictors of the Slope of the Level 1 Predictor and Criterion } \\
\hline & Social Contact (1, Alone; 2, With Others), $\gamma_{10}(d f=194)$ & Sex (Men, 1; Women, 2), $\gamma_{11}(d f=194)$ & Depression, $\gamma_{12}(d f=194)$ \\
\hline Positive Affect Index & $0.250(\mathrm{SE}, 0.035)^{* * *}$ & $0.007(\mathrm{SE}, 0.038)$ & $0.020(\mathrm{SE}, 0.041)$ \\
\hline Negative Affect Index & $-0.253(\mathrm{SE}, 0.031)^{* * *}$ & $-0.026(\mathrm{SE}, 0.027)$ & $-0.045(\mathrm{SE}, 0.033)$ \\
\hline Not well & $-0.141(\mathrm{SE}, 0.041)^{* *}$ & $-0.004(\mathrm{SE}, 0.039)$ & $-0.040(\mathrm{SE}, 0.046)$ \\
\hline \multirow[t]{2}{*}{ Ability to do activity } & $0.066(\mathrm{SE}, 0.038)$ & $0.061(\mathrm{SE}, 0.033)$ & $0.025(\mathrm{SE}, 0.050)$ \\
\hline & Closeness, $\gamma_{10}(d f=194)$ & Sex, $\gamma_{11}(d f=194)$ & Depression, $\gamma_{12}(d f=194)$ \\
\hline Positive Affect Index & $0.160(\mathrm{SE}, 0.009)^{* * *}$ & $-0.001(\mathrm{SE}, 0.010)$ & $0.025(\mathrm{SE}, 0.010)^{*}$ \\
\hline Negative Affect Index & $-0.065(\mathrm{SE}, 0.008)^{* * *}$ & $-0.004(\mathrm{SE}, 0.008)$ & $-0.034(\mathrm{SE}, 0.009)^{* * *}$ \\
\hline Not well & $-0.047(\mathrm{SE}, 0.013)^{* * *}$ & $-0.010(\mathrm{SE}, 0.011)$ & $0.034(\mathrm{SE}, 0.011)^{* *}$ \\
\hline Ability to do activity & $0.042(\mathrm{SE}, 0.012)^{* *}$ & $0.011(\mathrm{SE}, 0.009)$ & $0.026(\mathrm{SE}, 0.013)$ \\
\hline
\end{tabular}




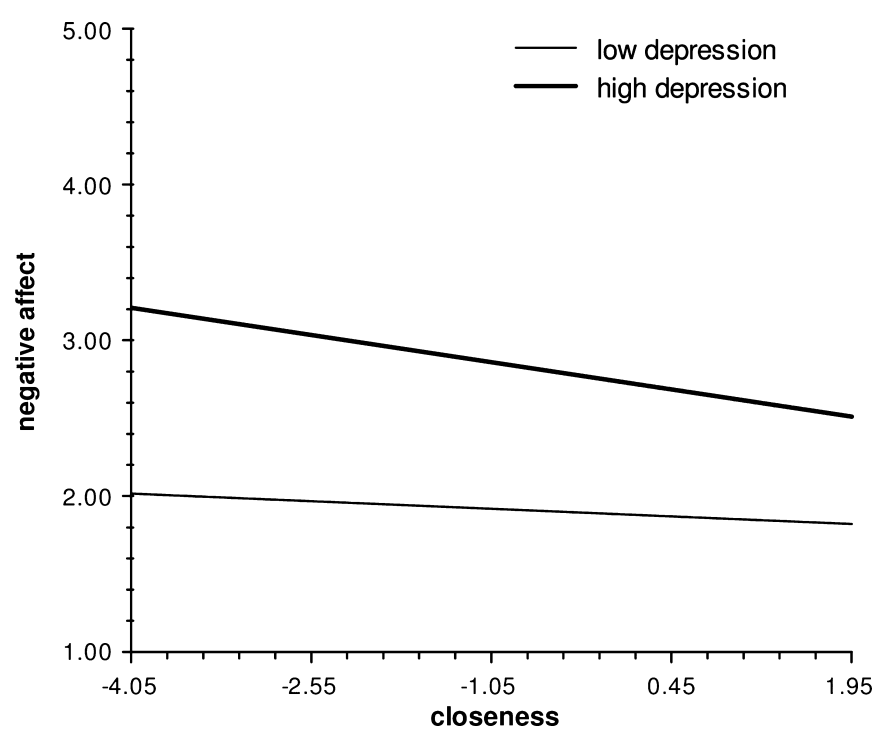

FIGURE 1. The cross-level interaction of depressive symptoms with negative affect and closeness.

of social interactions per se may influence the course of depressive symptoms. Coyne's (1976) interpersonal theory suggests that people with depressive symptoms demand excessive reassurance from others, which eventually alienates those around them and causes a long-term decline in social support. One possible explanation for our findings, viewed within the context of this theory, is that participants with depressive symptoms perceived people who provided reassurance to be "close." Furthermore, the literature provides other mechanisms to explain the positive benefits of close relationships for those with depressive symptoms, such as adaptive inferential feedback (Panzarella et al., 2006). Future studies examining social interactions in the daily lives of those with depressive symptoms should attempt to examine specific mechanisms by which close relationships are beneficial.

There is a considerable discussion regarding whether psychopathology is best represented using a neo-Kraepelinian system of categorical disorders or using a dimensional approach (e.g., Krueger et al., 2005). The present study assessed depression as a dimensional construct, and the sample included participants whose scores fell from the nondepressed to the severely depressed range. The findings provided support for a growing body of research suggesting that nonclinical levels of depression are associated with impairments in several domains of life functioning.

The findings that depressive symptoms were associated with reports of impairment in affect and functioning in daily life support the validity of ESM. The findings also suggest the utility of ESM for basic, translational, and applied research on depression. In particular, the method could help address some of the fundamental questions about the nature of clinical depression and provide essential basic data on how depression influences social interactions. For example, ESM research might allow researchers to examine "causal" chains of symptom patterns; for example, examining the primacy of affect versus cognition in the experience of depressive symptoms. Furthermore, given that future diagnostic models of depression-such as those that will be included in the DSM-V-will likely incorporate dimensional approaches, ESM studies may be useful in clarifying diagnostic criteria. Likewise, ESM may offer opportunities to deliver and assess the efficacy of treatment in the real world.

\section{CONCLUSIONS}

The present study has a number of potential clinical implications. It supports not the clinical interventions that aim just to increase behavior but those that specifically aim to increase supportive social contact (Harris et al., 1999). Furthermore, the present findings suggest that the closeness of the person with whom one is interacting could be important in determining whether the social contact will be beneficial, adding a different perspective to traditional behavioral activation theory for depression treatment. However, given that people with depression may be less likely to feel close to people, the development of treatment modules specifically targeted to increase relationship closeness could be a beneficial addition to existing treatment packages. Specifically, future studies should examine whether behavioral activation interventions may be most effective when specifically facilitating increased social interactions with close friends or family members. The present
A

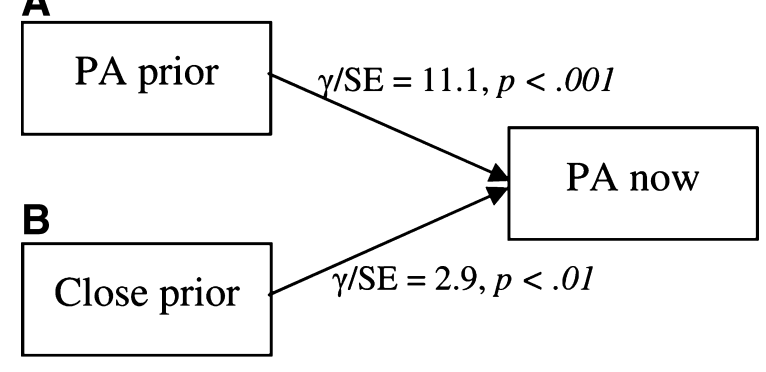

E

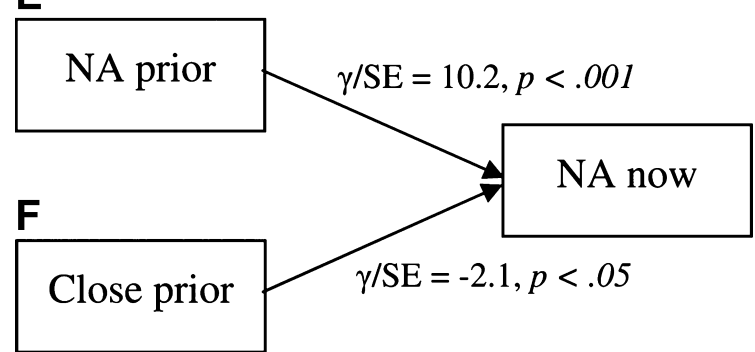

C

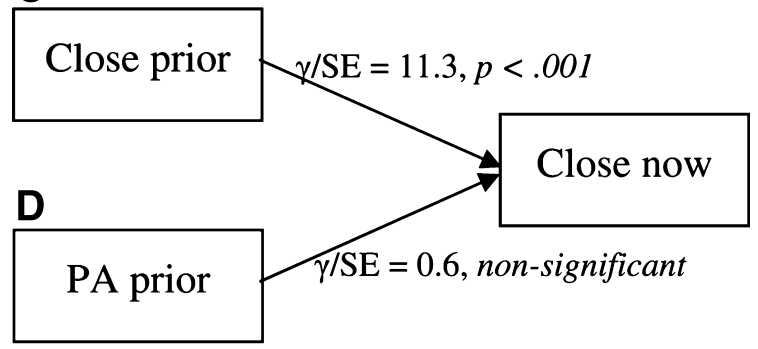

G

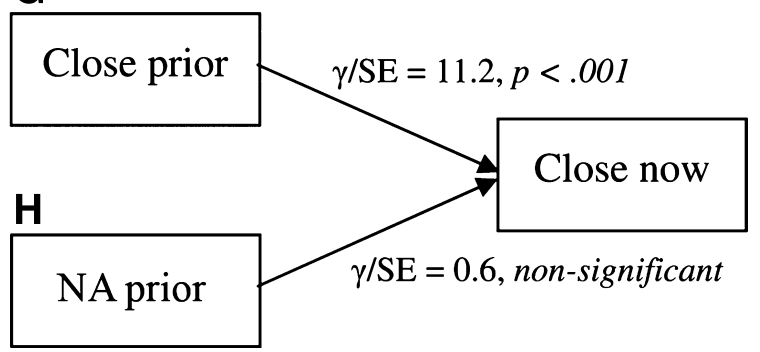

FIGURE 2. Association of previous ratings of affect and closeness with current ratings of affect and closeness. PA indicates positive affect; NA, negative affect. 
study was limited by using a simple (1-item) measure of closeness. Future studies should examine what relationship characteristics (e.g., trust, support, familiarity) drive the protective effects of closeness. In summary, ESM appears to provide a useful method for exploring the nature and expression of depressive symptoms and may be a useful vehicle for delivering and assessing the treatment of depression.

\section{ACKNOWLEDGMENT}

The authors thank Inez Myin-Germeys for her contributions.

\section{REFERENCES}

Airaksinen E, Wahlin A, Larsson M, Forsell Y (2006) Cognitive and social functioning in recovery from depression: Results from a population-based three-year follow-up. J Affect Disord. 96:107-110.

American Psychiatric Association (2004) Diagnostic and statistical manual of mental disorders (4th ed). Washington, DC: American Psychiatric Association.

Barge-Schaapveld D, Nicolson NA, Berkof J (1999) Quality of life in depression: Daily life determinants and variability. Psychiatry Res. 88:173-189.

Barnett PA, Gotlib IH (1988) Psychosocial functioning and depression: Distinguishing among antecedents, concomitants, and consequences. Psychol Bull. 104:97-126.

Beck AT, Ward CH, Mendelson M, Mock J, Erbaugh J (1961) An inventory for measuring depression. Arch Gen Psych. 4:561-571.

Beck, AT (1983) Cognitive therapy of depression: New perspectives. In P Clayton, JE Barrett (Eds), Treatment of depression: Old controversies and new approaches (pp 265-290). New York: Raven Press.

Billings AG, Moos RH (1984) Coping, stress, and social resources among adults with unipolar depression. J Pers Soc Psychol. 46:877-891.

Brown GW, Bifulco A, Harris T, Bridge L (1986) Life stress, chronic subclinica symptoms and vulnerability to clinical depression. J Affect Disord. 11:1-19.

Chepenik LG, Have TT, Oslin D (2006) A daily diary study of late-life depression. Am J Geriatr Psychiatry. 14:270-279.

Cohen S, Wills TA (1985) Stress, social support, and the buffering hypothesis. Psychol Bull. 98:310-357.

Coyne JC (1976). Toward an interactional description of depression. Psychiatry. 39: $28-40$.

Coyne JC, Whiffen VE (1995) Issues in personality as a diathesis for depression: The case of sociotropy-dependency and autonomy-self-criticism. Psychol Bull. 118:358-378.

Csikszentmihalyi M, Larson R (1987) Validity and reliability of the ExperienceSampling Method. J Nerv Ment Dis. 175:526-536.

Fergusson DM, Boden JM, Horwood LJ (2007) Recurrence of major depression in adolescence and early adulthood, and later mental health, educational and economic outcomes. Br J Psychiatry. 191:335-342.

Hankin BL, Fraley RC, Lahey BB (2005) Is depression best viewed as a continuum or discrete category? A taxometric analysis of childhood and adolescent depression in a population-based sample. J Abnorm Psychol. 114:96-110.
Harris T, Brown GW, Robinson R (1999) Befriending as an intervention for chronic depression among women in an inner city: Role of fresh-start experiences and baseline psychosocial factors in remission from depression. Br J Psychiatry. 174:225-232.

Hox J. Multilevel analysis: techniques and applications. Mahwah, NJ: Lawrence Erlbaum Associates; 2002.

Intel Corporation (2004) iESP [computer software]. Available at: http://seattleweb. intel-research.net/projects/ESM/iESP.html. Accessed September 1, 2004.

Judd LL, Paulus MP, Wells KB, Rapaport MH (1996) Socioeconomic burden of subsyndromal depressive symptoms and major depression in a sample of the general population. Am J Psychiatry. 153:1411-1417.

Kessler RC, Zhao S, Blazer DG, Swartz M (1997) Prevalence, correlates, and course of minor depression and major depression in the national comorbidity survey. $J$ Affect Disord. 45:19-30.

Klerman GL, Weissman MM, Rounsaville BJ, Chevron ES (1984) Interpersonal psychotherapy of depression. New York: Basic Books, Inc.

Kreft, I, de Leeuw J (1998) Introducing multilevel modeling. London: Sage.

Krueger RF, Markon KE (2006) Understanding psychopathology: Melding behavior genetics, personality, and quantitative psychology to develop an empirically based model. Curr Dir Psychol Sci. 15:113-117.

Krueger RF, Watson D, Barlow DH (2005) Introduction to the special section: Toward a dimensionally based taxonomy of psychopathology. J Abnorm Psychol. 114:491-493.

Lewinsohn PM, Hoberman HM, Rosenbaum M (1988) A prospective study of risk factors for unipolar depression. J Abnorm Psychol. 97:251-264.

Myin-Germeys I, van Os JJ, Schwartz JF, Stone AA, Delespaul PA (2001) Emotional reactivity to daily life stress in psychosis. Arch Gen Psychiatry. 58 $1137-1144$.

Panzarella C, Alloy LB, Whitehouse WG (2006) Expanded hopelessness theory of depression: On the mechanisms by which social support protects against depression. Cogn Ther Res. 30:307-333.

Peeters F, Berkhof J, Delespaul P, Rottenberg J, Nicolson NA (2006) Diurnal mood variation in major depressive disorder. Emotion. 6:383-391.

Raudenbush S, Bryk A, Congdon R (2004) HLM for Windows [computer software]. Version 6. Lincolnwood, IL: Scientific Software International

Reis HT, Gable SL (2000) Event-sampling and other methods for studying everyday experience. In HT Reis, CM Judd (Eds), Handbook of research methods in social and personality psychology (pp. 190-221). Cambridge, UK: Cambridge University Press.

Rusting CL, Larsen RJ (1998) Diurnal patterns of unpleasant mood: Associations with neuroticism, depression, and anxiety. J Pers. 66:85-103.

Schwartz JE, Stone AA (1998) Strategies for analyzing ecological momentary assessment data. Health Psychol. 17:6-16.

Scott J, Teasdale JD, Paykel ES, et al (2000) Effects of cognitive therapy on psychological symptoms and social functioning in residual depression. $\mathrm{Br} J$ Psychiatry. 177:440-446.

Wang, PS, Beck AL, Berglund P, McKenas DK, et al (2004) Effects of major depression on moment-in-time work performance. J Psychiatry. 161:1885-1891. 